REVESCO. Revista de Estudios Cooperativos

ISSN: $1885-8031$

https://dx.doi.org/10.5209/REVE.71866

\title{
Las comunidades energéticas en Grecia ${ }^{1}$
}

\author{
Gemma Fajardo García ${ }^{2}$ y Maria Frantzeskaki ${ }^{3}$
}

Recibido: 4 de julio de 2020 / Aceptado: 29 de septiembre de 2020 / Publicado: 26 de enero de 2021

Resumen. Grecia ha dado un importante impulso a la Economía Social y Solidaria a partir de 2011 con la aprobación de diversas normas que regulan este sector. Entre otras, y siguiendo de cerca los trabajos llevados a cabo por las instituciones europeas, Grecia ha regulado por vez primera en Europa, las comunidades energéticas. Estas se definen como cooperativas civiles que tienen como objeto exclusivo promover la economía social y solidaria y la innovación en el sector energético; la lucha contra la pobreza energética y la promoción de la energía sostenible, la producción, almacenamiento, autoconsumo, distribución y suministro de energía; aumentar la autosuficiencia energética y la seguridad en los municipios de las islas; así como la mejora de la eficiente energética en el uso final, en el ámbito local y regional, a través de sus actividades en el sector de las fuentes de energía renovable, la cogeneración de energía eléctrica de alta eficiencia, del uso racional de la energía, de la eficiencia energética, del transporte sostenible, de la gestión de la demanda y la producción, distribución y suministro de energía. En este trabajo hemos querido conocer esa regulación pionera de las comunidades energéticas, la problemática que genera su concepción como cooperativa, los diversos modelos de comunidades energéticas que podrían desarrollarse de conformidad con su exclusivo objeto social, y las experiencias que se han dado hasta el momento, así como sus principales características.

Palabras clave: Cooperativas; Electricidad; Legislación; Experiencias; Unión Europea.

Claves Econlit: L3; K23; O180; Q48.

\section{[en] The energy communities in Greece}

\begin{abstract}
Greece has given an important boost to the Social and Solidarity Economy since 2011 with the approval of various laws that regulate this sector. Among others, and closely following the work carried out by the European institutions, Greece has regulated for the first time in Europe, the energy communities. These are defined as civil cooperatives whose sole purpose is to promote the social and solidarity economy and innovation in the energy sector; the fight against energy poverty and the promotion of sustainable energy, production, storage, self-consumption, distribution and supply of energy; increase energy self-sufficiency and security in the municipalities of the islands; as well as the improvement of energy efficiency in end use, locally and regionally, through its activities in the sector of renewable energy sources, the cogeneration of highly efficient electrical energy, the rational use of energy , energy efficiency, sustainable transport, demand management and the production, distribution and supply of energy. In this work we wanted to learn about know this pioneering regulation of energy communities, the problems generated by its conception as a cooperative, the various models of energy communities that could be developed in accordance with its exclusive corporate purpose, and the experiences that have been given so far, as well as its main characteristics.
\end{abstract}

Keywords: Cooperatives; Electricity; Legislation; Experiences; European Union.

Sumario. 1. Introducción. 2. El impulso a la Economía Social y Solidaria en Grecia en los últimos años. 3. Las entidades de la economía social y solidaria en Grecia. 4. El reconocimiento y regulación de las comunidades energéticas en la Ley 4513/2018. 5. Modelos de comunidades energéticas en Grecia. 6. Experiencias de comunidades energéticas en Grecia. 7. Financiación de las comunidades energéticas. 8. Conclusiones. 9. Referencias bibliográficas.

Cómo citar. Fajardo García, G.; Frantzeskaki, M. (2021) Las comunidades energéticas en Grecia. REVESCO. Revista de Estudios Cooperativos, vol. 137, e71866. https://dx.doi.org/10.5209/reve.71866.

1 Este artículo desarrolla y actualiza la comunicación presentada por las autoras en el XVII Congreso Internacional de Investigadores en Economía Social. La Economía Social: transformaciones recientes, tendencias y retos de futuro, organizado por Ciriec-España y celebrado en Toledo, el 4 y 5 de octubre de 2018. Y es resultado del proyecto de investigación: Proyecto I+D+i: Economía Social, Autogestión y Empleo (DER2016-78732$\mathrm{R})$, financiado por el $\mathrm{M}^{\mathrm{o}}$ de Ciencia, Innovación y Universidades, Agencia Estatal de Investigación y Fondo Europeo de Desarrollo Regional (FEDER).

2 Universitat de Valencia, España.

Dirección de correo electrónico: fajardo@uv.es.

3 Universitat de Valencia, España.

Dirección de correo electrónico: mafrant@alumni.uv.es 


\section{Introducción}

Grecia viene promoviendo desde 2011 la Economía Social y Solidaria dando un claro impulso a todas estas entidades, mediante leyes que buscan transformar la economía helena con una orientación más social y sostenible. Como ejemplo de este impulso cabe señalar la Ley 4513/2018, de 22 de enero, sobre Comunidades Energéticas, con la que se regula por vez primera en Europa este modelo de organización, que en Grecia debe adoptar la forma jurídica de cooperativa, y con la que se pretende promover la innovación en el sector energético, luchar contra la pobreza energética y promover la energía sostenible, tanto su producción, como su almacenamiento, autoconsumo y distribución. La experiencia de Grecia es en estos momentos un referente para toda Europa y por ello las autoras de este trabajo, hemos querido conocer mejor, no sólo su pionera regulación, sino también su implantación en el país.

En las siguientes páginas vamos a esbozar someramente el marco normativo griego en materia de economía social, cooperativismo y empresas sociales, como previo al análisis de la citada Ley 4513/2018 sobre Comunidades Energéticas. Previamente, haremos referencia a los trabajos en curso elaborados por las instituciones europeas en torno al reconocimiento de las comunidades de energía renovable y comunidades locales, y que inspiraron en su momento al legislador griego. Una vez analizado el contenido de la ley nos cuestionamos la compatibilidad entre la comunidad energética y la forma cooperativa elegida, tal y como se concibe en Grecia.

Diseñaremos los diversos modelos de comunidades energéticas que el exclusivo objeto social previsto permite y por último, analizaremos las experiencias de comunidades energéticas existentes en este momento en Grecia, a la vista de los datos ofrecidos por las instituciones públicas, de sus normas internas de funcionamiento y de sus principales rasgos característicos.

\section{El impulso a la Economía Social y Solidaria en Grecia en los últimos años}

La economía social y solidaria ha sido objeto de un importante impulso en Grecia desde los años 2011 con la aprobación de la Ley 4019/2011, de 30 de septiembre de "Economía Social y Emprendimiento Social", y más tarde de la Ley 4430/2016, de 31 de octubre de "Economía Social y Solidaria" que deroga la anterior y amplia el ámbito de la economía social en aquel país, regulando en particular las cooperativas de trabajadores. Estas normas se han visto complementadas más tarde, con la Ley 4384/2016, de 26 de abril de Cooperativas Agrarias; la Ley 4423/2016, de 27 de septiembre, de Cooperativas Forestales; la Ley 4513/2018, de 22 de enero, sobre Comunidades Energéticas que regula las cooperativas energéticas, objeto central de este estudio, y la más reciente Ley 4600/2019, de 8 de marzo, que regula las llamadas Cooperativas de Integración en el Sector de la Salud. Más ampliamente sobre el marco jurídico que configura la Economía social y solidaria en Grecia en Fajardo y Frantzeskaki (2017).

La economía social y solidaria se define en Grecia como un conjunto de actividades económicas caracterizadas por una forma alternativa de organización de las relaciones de producción, distribución, consumo y reinversión, basada en los principios de democracia, igualdad, solidaridad, cooperación y respeto al ser humano y al medio ambiente (art. 2.1 Ley 4430/2016).

\section{Las entidades de la economía social y solidaria en Grecia}

La Ley 4430/2016 no sólo define la economía social y solidaria, sino que identifica lo que denomina entidades de la economía social y solidaria, y lo hace siguiendo un doble criterio, por su forma o calificación jurídica, y por concurrir en las mismas ciertas características. Son entidades de la economía social y solidaria por su forma o calificación jurídica, las que se constituyen como Empresas Sociales Cooperativas, Cooperativas Sociales de Responsabilidad Limitada o Cooperativas de Trabajadores. Pero también podrán considerarse entidades de la economía social y solidaria otras personas jurídicas, en especial -dice- las cooperativas agrarias y civiles, y las sociedades civiles, que cumplan ciertas condiciones: a) que sus actividades reporten un beneficio común o social; b) que apliquen un sistema democrático en la toma de decisiones; c) que no distribuyan beneficios entre sus miembros (a excepción de los trabajadores); d) que la horquilla salarial no supere tres veces el salario mínimo y e) que cooperen con otras entidades de la economía social y solidaria y no dependan directa ni indirectamente de entidades públicas.

La Comunidad Energética, creada con la Ley 4513/2018 no se concibe como un nuevo modelo de entidad de la economía social y solidaria por la forma, sino que se concibe como un modelo más de cooperativa civil, y, por lo tanto, adquirirá aquella condición en la medida en que cumpla con los anteriores requisitos.

El concepto de cooperativa civil alude a la clásica distinción entre cooperativas rurales (actualmente agrícolas) y urbanas o civiles, presente en la Constitución Helena de 1975. Son cooperativas civiles todas aquellas que no son cooperativas agrarias. Su regulación se contiene básicamente en la Ley General de 
Cooperativas 1667/1986, de 5 de diciembre, que es de aplicación a todas las cooperativas que no tengan una ley especial, y con carácter supletorio, en los demás casos. La concepción de la cooperativa presente en esta ley responde al modelo mutualista; así se define como una unión voluntaria de personas que mediante la cooperación llevan a cabo actividades económicas para el desarrollo económico, social y cultural de sus miembros, y la mejora de su calidad de vida, generalmente en el marco de una empresa común (art. 1.1).

\section{El reconocimiento y regulación de las comunidades energéticas en la Ley 4513/2018}

En 2018 Grecia aprobó la ley por la que regula las comunidades energéticas, siendo así, el primer país en Europa en regular esta entidad; pero para entender el objetivo de esta ley y la configuración de estas comunidades, debe tenerse en cuenta también el entorno socio jurídico en que se gesta esta norma. Es por tanto necesario hacer una mención, aunque sea breve, a los trabajos pre-legislativos que en ese momento se estaban llevando a cabo en las instituciones europeas para regular las comunidades energéticas.

\subsection{Antecedentes: la comunidad local de energía y la comunidad de energía renovable, en la Unión Europea}

Ifigenia Douvitsa (2018:33) señala dos circunstancias como desencadenantes de la situación crítica que llevó al Ministro de Desarrollo y Energía griego a promover la regulación de las comunidades energéticas. Por una parte, los elevados índices de pobreza energética en $\mathrm{Grecia}^{4}$, y por otra, la situación de muchas islas del Mar Egeo, sin conexión a la infraestructura eléctrica continental y por tanto, totalmente dependientes de combustibles fósiles con lo que tienen que hacer frente a elevados costes de energía. A todo ello hay que añadir, los trabajos que desde las instituciones europeas se venían llevando a cabo para regular y promover el autoconsumo de energía renovable entre los ciudadanos, y en los que sin duda se inspiró el Gobierno griego a la hora de elaborar la Ley 4513/2018 (Douvitsa 2018: 36).

Los antecedentes de la regulación de las comunidades energéticas (CCEE) en Grecia hay que situarlos por tanto en la normativa de la Unión Europea y en particular, en la Comunicación de la Comisión "Energía limpia para todos los europeos" de 30 de noviembre de 2016 [COM(2016) 860 final] y en las propuestas de directivas que en desarrollo de la anterior se habían aprobado en 2017. La citada Comunicación aprobó un amplio paquete de medidas con las que se pretendía liderar el mercado de las energías renovables, y poner a los consumidores en el centro de la Unión de la Energía reivindicando para éstos un trato más justo, de forma que pudieran "producir su propia energía, almacenarla, compartirla, consumirla o venderla en el mercado, directamente o a través de cooperativas de energía". Algunas de esas medidas se concretaron en dos propuestas de directivas publicadas el 23 de febrero de 2017: una sobre normas comunes para el mercado interior de la electricidad [COM(2016 864 final], y otra sobre fomento del uso de energía procedente de fuentes renovables [COM(2016) 767 final]. La primera regulaba en su artículo 16 las "Comunidades Locales de Energía", que definía como entidades con personalidad jurídica, controladas por miembros locales, generalmente orientadas al valor más que a la rentabilidad, y dedicadas a la generación, distribución, agregación, almacenamiento, suministro de energía o prestación de servicios de eficiencia energética, en el ámbito local. La segunda hacía referencia en cambio, a las "Comunidades de energía renovable", que definía como aquellas, cuyos miembros colaboran en la generación, distribución, almacenamiento o suministro de energía procedente de fuentes renovables y que además, cumplen determinados requisitos: $1^{\circ}$ que al menos el $51 \%$ de los puestos del consejo de administración correspondan a miembros locales (representantes de los intereses socioeconómicos locales públicos y privados, o ciudadanos particulares), y $2^{\circ}$ que cumpla al menos tres de los siguientes requisitos: a) que sus partícipes o miembros sean personas físicas, autoridades locales (incluidos los municipios) o pymes; b) que al menos el $51 \%$ de los miembros con derecho a voto sean personas físicas u organismos públicos; c) que al menos el $51 \%$ de las participaciones de la entidad pertenezcan a miembros locales, y d) que la comunidad no haya instalado más de $18 \mathrm{MW}$ de capacidad de energías renovables de media anual en los cinco años anteriores. Más ampliamente sobre el contenido y orientación de estas propuestas de directiva en Fajardo (2019).

Estas propuestas de directivas fueron reformuladas más tarde y aprobadas como Directiva (UE) 2019/944 y Directiva (UE) 2018/2001, del Parlamento y del Consejo de 5 de junio de 2019 sobre normas comunes para el mercado de la electricidad (DMIE en lo sucesivo), y, de 11 de diciembre de 2018 relativa al fomento del uso de energía procedente de fuentes renovables (DFER en lo sucesivo), respectivamente. Ambas directivas contemplan las comunidades energéticas como expresión de la participación de los consumidores en el mercado de la energía, a través de entidades constituidas para tal fín. La DFER conserva la denominación de Comunidades de Energía Renovable (art. 2.16) mientras que la DMIE ha sustituido la denominación de 
"Comunidades Locales de Energía" por la de "Comunidades ciudadanas de energía" (art. 2.11). Ambas son objeto de regulación separadamente, aunque con muchos puntos de coincidencia (RESCoop, 2020: 10). Para conocer el régimen jurídico de estas comunidades debe tenerse en cuenta tanto su regulación en la DFER (art. 22) como en la DMIE (art. 16).

De la conjunción de ambos ordenamientos cabe señalar como características propias de una Comunidad Energética (CE) siguiendo a Fajardo y Talaverano (2020) las siguientes:

a) En primer lugar, se trata de una nueva entidad, con personalidad jurídica, cualquiera que sea su forma, siempre que de acuerdo al derecho nacional aplicable se base en la participación abierta y voluntaria de sus socios o miembros, y sea autónoma. La proposición de DFER concretaba que la comunidad fuera una pyme o una organización sin ánimo de lucro, pero el texto finalmente aprobado no incorpora ninguna categoría en particular, siendo en principio cualquiera que según la legislación nacional se base en la participación abierta y voluntaria de sus socios o miembros, y que sea autónoma. En particular se quiere que las comunidades sean accesibles a todos los consumidores, incluidos aquellos con ingresos bajos o vulnerables (art. $22.4 \mathrm{f}$ DFER) y que cualquier socio o miembro tenga derecho a abandonar la comunidad cuando quiera (art. 16. 1 b DMIE).

b) Sus socios o miembros deben ser personas físicas, pymes o autoridades locales (como municipios). La comunidad ciudadana de energía admite otros socios o miembros siempre que el control efectivo de la comunidad esté en manos de los anteriores (art. 2.11 DMIE). La directiva no exige un número mínimo de miembros/socios personas físicas, a diferencia de la Proposición que exigía que al menos un $51 \%$ de los miembros de la entidad con derecho de voto fueran personas físicas (art. $22.1 \mathrm{~b}$ ). Y tampoco exige que el control de la entidad esté en manos de miembros locales como exigía la proposición (art. 22.1. c y d) sin embargo la DFER si que exige que los socios o miembros de la CE estén situados en las proximidades de los proyectos de energía renovable (art. 2.16).

c) La finalidad primordial de la comunidad ha de ser proporcionar beneficios medioambientales, económicos o sociales a sus socios o miembros o a las zonas locales donde opera (o localidad en la que desarrolla su actividad, según la DMIE), en lugar de ganancias financieras. Obsérvese que el objeto social se plantea como opcional: o proporciona beneficios a sus socios o a la comunidad. No se entiende por qué y deberá interpretarse como posible y deseable, que la entidad comunitaria proporcione esos beneficios tanto a sus miembros o socios, como a la comunidad.

d) A las anteriores características, la DMIE añade que la comunidad participa en la generación, incluida la procedente de fuentes renovables, la distribución, el suministro, el consumo, la agregación, el almacenamiento de energía, la prestación de servicios de eficiencia energética o, la prestación de servicios de recarga para vehículos eléctricos, u otros servicios energéticos a sus miembros o socios.

e) Por último, la vigente normativa no pone límite a la capacidad máxima de las instalaciones de la comunidad, a diferencia de la proposición (art. 22.1.e).

Así mismo, la legislación ordena a los Estados que evalúen los obstáculos existentes y el potencial de desarrollo de las comunidades en sus territorios, y que proporcionen un marco jurídico que permita fomentar y facilitar su desarrollo.

Como principales novedades cabe destacar:

a) La posibilidad que se reconoce a las comunidades de compartir en su seno la energía renovable que produzcan las unidades de producción propiedad de dicha comunidad (art. 22. 2 b DFER); a tal fin se exige que el gestor de la red de distribución correspondiente coopere con las comunidades de energías renovables para facilitar, en el seno de las comunidades de energías renovables, las transferencias de energía (art 22.4 c DFER).

b) En la misma línea, la DMIE ordena a los Estados que creen un marco jurídico favorable que reconozca a las comunidades el derecho a poseer, establecer, adquirir o arrendar redes de distribución y gestionarlas autónomamente (art. 162 b).

c) En todas las actividades que desarrollen estas comunidades como consumidores finales, generadores, suministradores, gestores de redes de distribución o participantes en el mercado que presten servicios de agregación, se beneficiarán de un trato no discriminatorio y proporcionado (art. 16.4 b DMIE)

d) En el marco facilitador que el Estado debe proporcionar para el desarrollo de las comunidades, debe poner a disposición de éstas información, y debe facilitarse su acceso a la financiación. Así mismo, debe proporcionarse apoyo reglamentario y de refuerzo de capacidades a las autoridades públicas para propiciar y crear CCEE, así como para ayudar a las autoridades a participar directamente en ellas (art. $22.4 \mathrm{~g}$ y h DFER). 


\subsection{Concepto y marco jurídico aplicable a las comunidades energéticas en Grecia}

Las comunidades energéticas griegas se regulan en la Ley 4513/2018, de 22 de enero sobre Comunidades Energéticas. Ley que se ha adelantado a la entrada en vigor de las Directivas europeas y con la que el Gobierno griego pretende facilitar la transición del país a la energía verde; promover que los ciudadanos, los municipios y las empresas locales participen en la transición energética y la planificación energética a través de su participación activa directa; abordar el problema de la pobreza energética, y favorecer la autonomía energética de las regiones insulares.

La comunidad energética (CE) se describe en esta Ley como una cooperativa civil que tiene como objeto exclusivo promover la economía social y solidaria y la innovación en el sector energético; la lucha contra la pobreza energética y la promoción de la energía sostenible, la producción, almacenamiento, autoconsumo, distribución y suministro de energía; aumentar la autosuficiencia energética y la seguridad en los municipios de las islas; así como la mejora de la eficiente energética en el uso final, en el ámbito local y regional, a través de sus actividades en el sector de las fuentes de energía renovable, la cogeneración de energía eléctrica de alta eficiencia, del uso racional de la energía, de la eficiencia energética, del transporte sostenible, de la gestión de la demanda y la producción, distribución y suministro de energía (art. 1).

Siendo la comunidad energética un modelo de cooperativa civil, habrá que tener en cuenta que la misma se regirá además de por la ley que analizamos, por la ley 1667/1986 que regula las cooperativas civiles. En materia de fomento en cambio, se extiende a las CCEE la normativa contenida en la Ley 4399/2016 aplicable a las empresas sociales cooperativas (art. 11.1).

Se ha criticado con razón que, siendo la CE una cooperativa, no adopte esta denominación, lo cual generará confusión sobre su identidad jurídica. Por otra parte, debe tenerse en cuenta que en Grecia es posible constituir una cooperativa energética también bajo la Ley 1667/1986 que regula las cooperativas civiles y bajo la Ley 4430/2016 que regula las cooperativas sociales y cooperativas de trabajadores (Douvitsa, 2018: 43).

\subsection{El objeto social de las comunidades energéticas}

Elemento clave para comprender las CCEE es conocer el ámbito de su objeto social. La ley determina en concreto cuales son las actividades que pueden integrar ese objeto social con carácter exclusivo, no permitiendo que los estatutos sociales incorporen otras distintas, lo que posiblemente limitará la funcionalidad del modelo. La CE debe realizar al menos una de las actividades previstas en el art. 4.1, y puede, además, llevar a cabo otras de las contempladas en el art. 4.2, si así se prevé estatutariamente.

Tabla. 1. Actividades propias y complementarias de las comunidades energéticas.

\section{Actividades propias de las CCEE (art. 4.1):}

a) La producción, almacenamiento, consumo propio o venta de energía eléctrica o térmica o de refrigeración de plantas de fuentes de energía renovables o de cogeneración de energía eléctrica de alta eficiencia, o plantas híbridas establecidas en la región donde se encuentra la sede de la C.E. o incluso dentro de la región vecina de la C.E. con sede en la región de Attica.

b) Las de gestión, como recogida, transporte, procesamiento, almacenamiento o suministro, de materia prima para la producción de energía eléctrica o térmica o de refrigeración a partir de biomasa, biolíquidos o biogás, o mediante la recuperación de energía de la fracción biodegradable de los residuos municipales.

c) Procurar para sus miembros los productos energéticos, aparatos e instalaciones que precisen, con el objetivo de reducir el consumo de energía y el uso
Actividades complementarias que pueden integrarse en el objeto social de la CE (art. 4.2):

a) La captación de fondos para inversiones en la explotación de fuentes de energía renovables o de cogeneración de energía eléctrica de alta eficiencia, o intervenciones para mejorar la eficiencia energética dentro de la región donde se encuentra la sede de la C.E.

b) Compilación de estudios de utilización de fuentes de energía renovables o de cogeneración de energía eléctrica de alta eficiencia, o la implementación de intervenciones de mejora de la eficiencia energética o la prestación de asistencia técnica a los sectores antes mencionados.

c) Gestionar o participar en programas financiados con recursos nacionales o de la Unión Europea con respecto a sus fines.

d) Asesorar sobre la gestión o la participación de sus miembros en programas financiados con 
de combustible, así como mejorar la eficiencia energética.

d) Procurar para sus socios vehículos motorizados eléctricos, híbridos o no híbridos y en general, vehículos que utilizan combustibles alternativos.

e) La distribución de electricidad dentro de la región donde se encuentra su sede.

f) El suministro de electricidad o gas natural a clientes finales dentro de la región en la que se encuentra su sede.

g) La producción, distribución y suministro de energía térmica o de refrigeración dentro de la región donde se encuentra su sede,

h) La gestión de la demanda para reducir el uso final de la energía eléctrica y la representación de productores y consumidores en el mercado de la electricidad.

i) El desarrollo de una red, la gestión y explotación de la infraestructura de combustibles alternativos o la gestión de medios de transporte sostenible dentro de la Región donde se encuentra la sede de la C.E.

j) La instalación y funcionamiento de plantas de desalinización utilizando fuentes de energía renovables dentro de la región donde se encuentra la sede de la C.E.

k) La prestación de servicios energéticos. fondos nacionales o de la Unión Europea con respecto a sus propósitos.

e) La información, educación y sensibilización a nivel local y regional sobre cuestiones de sostenibilidad energética

f) La realización de acciones para apoyar a los consumidores vulnerables y afrontar la pobreza energética de las personas que viven por debajo de los límites de pobreza de la región en la que se encuentra la sede de la C.E., independientemente de si son miembros de C.E., como el suministro o la compensación de energía, mejora energética de los hogares, u otras acciones que reducen el consumo de energía en sus hogares.

Fuente: Elaboración propia basada en la Ley 4513/2018.

\subsection{Los miembros de las comunidades energéticas. Clases y requisitos}

Los miembros de las CCEE pueden ser: personas físicas o jurídicas, de derecho público o privado, y autoridades locales y regionales, así como sus empresas. Ningún miembro puede pertenecer a más de una $\mathrm{CE}$, salvo que se trate de una persona jurídica pública o de una autoridad local.

Para constituir una CE se requiere la presencia mínima de cinco miembros, sean personas físicas o jurídicas; siendo suficiente con tres miembros si dos de ellos al menos son autoridades locales; o de dos miembros si son autoridades locales de primer grado, en regiones insulares con una población inferior a 3.100 habitantes.

En todo caso se requiere que el $50 \%$ más uno de los miembros estén vinculados con el lugar donde se encuentra la sede de la CE; esto es, en el caso de las personas físicas, deben tener bienes raíces en propiedad o arrendamiento en la región de la sede de la CE o tener su residencia en dicha región, y en el caso de las personas jurídicas, deben tener su domicilio social en la región de la CE.

La posible presencia de entidades públicas como miembros de una cooperativa constituye una importante novedad en la legislación cooperativa griega, ya que hasta ahora su presencia no era posible; sin embargo, se ha justificado teniendo en cuenta que la energía es un bien común y que las entidades públicas pueden aportar recursos personales y económicos en su desarrollo (Douvitsa 2018: 45).

\subsection{La constitución de comunidades energéticas}

Como hemos visto, las CCEE se constituyen como cooperativas civiles. Al registrarse, deberán presentar sus estatutos sociales, y en el caso de contar con miembros personas físicas, las certificaciones que acrediten la ubicación de sus bienes raíces o su residencia. 
En los estatutos de la CE se hará constar al menos su denominación, que debe incluir el término "Comunidad Energética" o su abreviatura "C.E." así como la indicación sobre el alcance de la responsabilidad de sus miembros; se identificarán éstos, el propósito perseguido y las actividades previstas; los requisitos de entrada, salida y exclusión de sus miembros; sus responsabilidades, derechos y obligaciones, y consecuencias de su incumplimiento; domicilio de la CE, que podrá ser un municipio o un distrito municipal; su duración o el número de miembros de su órgano de administración, que no podrá ser inferior a tres.

La CE adquiere su personalidad jurídica cuando se inscribe en el Registro de CCEE del Registro General Mercantil, sin ser necesario su inscripción en ningún otro registro, ni siquiera en el de cooperativas. El Registro General Mercantil se ocupa también de publicar la información financiera de la CE (balance, cuenta de pérdidas y ganancias, informe del órgano de administración y de los auditores) una vez aprobada por su asamblea general.

\subsection{La aportación o cuota cooperativa y la distribución de excedentes}

Especial atención debe hacerse a otras menciones que deben contener los estatutos sociales como son las cuotas cooperativas y el destino de los excedentes del ejercicio.

4.6.1. Los estatutos deben indicar el importe de la cuota a abonar para ser cooperativista (cuota cooperativa), la forma y momento de su pago, el proceso de devolución y su destino en caso de fallecimiento del miembro.

Cada socio puede tener además de la cuota cooperativa obligatoria, una o más cuotas voluntarias, siempre que no excedan del $20 \%$ del capital, excepto si se trata de una autoridad local que puede llegar a tener una participación en el capital del $40 \%$ e incluso del $50 \%$ si se trata de una autoridad local de primer grado de regiones insulares con una población de menos de 3.100 habitantes.

Las cuotas cooperativas son transmisibles tanto a miembros como a terceros, pero previo acuerdo de la asamblea general, y siempre que no se superen los límites que establece la ley. En todo caso, con independencia del número de cuotas que tenga un miembro de una CE, su voto será único (art. 3).

4.6.2. Los estatutos también deben indicar la forma de disposición de los excedentes de cada ejercicio de la CE, dentro de los límites que marca la Ley. Debe tenerse en cuenta que la ley que comentamos denomina "excedente" a todo resultado positivo, sin distinguir como es propio en la cooperativa entre, los resultados de las operaciones con socios (excedentes/surpluses), de los obtenidos en otras operaciones (beneficios/profits) (Kaminari, O., Papageorgiou, K., 2016); esta no distinción tampoco permite dar a cada resultado el destino que correspondería en una cooperativa, como subraya Douvitsa (2018: 50). La ley establece que al menos el $10 \%$ de los "excedentes", deben retenerse para conformar la reserva ordinaria de la CE hasta que ésta alcance un importe igual al del capital social.

Los demás excedentes podrán destinarse según indica el art. 6:

a) A ser distribuidos entre los socios en el caso en que se den los siguientes requisitos:

- que la CE tenga al menos 15 socios, o 10 socios si se trata de una CE con sede en una isla con población de menos de 3.100 habitantes;

- que el 51\% de los socios, al menos, sean personas físicas (condición que debe cumplirse en todo momento desde la constitución de la CE);

- que los estatutos hayan previsto la distribución del excedente.

b) A reservas a disposición de la asamblea general para la realización de sus fines.

c) A actuaciones de interés general de importancia local y relacionadas con la suficiencia y el suministro de materias primas, combustible y agua, aunque no sean los fines propios de la CE si:

- en la CE participan sólo autoridades locales de primer o segundo grado de la región en la que se encuentra la sede de la CE,

- si la CE tiene su sede en un municipio insular con una población de menos de 3.100 habitantes y cuenta con una autoridad local de primer o segundo grado de la región en la que se encuentra la sede de la CE.

\subsection{Disolución, liquidación y modificaciones estructurales de las comunidades energéticas}

La disolución de la CE tendrá lugar cuando se cumpla el plazo previsto en los estatutos, se superen los límites exigidos en la ley, se declare la quiebra de la CE o su asamblea general así lo acuerde.

Una vez disuelta la CE se procederá a su liquidación y una vez pagadas las deudas pendientes, el saldo positivo resultante se destinará: en primer lugar, a devolver a los miembros sus cuotas cooperativas obligatorias y voluntarias, y el resto se distribuirá a las comunidades de productores, asociaciones de 
personas, organizaciones y personas jurídicas sin ánimo de lucro del sector de la energía y protección del medio ambiente de la región en la que se encuentre la sede de la CE. Como excepción, las CCEE que cumplen los requisitos que les permiten distribuir los excedentes disponibles entre sus socios, como vimos en el apartado 4.6.2 a, deben distribuir el saldo restante a estos, en proporción a su participación en el capital social (art. 9.2). Sorprende que el legislador cree una entidad jurídica que debe adoptar la forma de cooperativa, pero debe regirse por normas propias de las sociedades de capital, o como se pregunta Douvitsa, (2018: 52) ¿por qué el legislador da la forma jurídica de cooperativa a una CE orientada a la obtención de beneficios?

Las CCEE pueden fusionarse entre sí siempre que apliquen sus excedentes de forma similar y sus sedes se ubiquen en la misma región. También contempla la Ley la posibilidad de conversión de los distintos tipos de cooperativas en CCEE.

\section{8. Incentivos financieros y medidas de apoyo}

La Ley finalmente establece un amplio paquete de medidas de apoyo a las CCEE, relativas tanto a su financiación como a su actividad. Entre otras se le reconocen privilegios en la contratación pública de servicios, en la concesión de licencias de producción de energía y en la transferencia de éstas.

De especial interés es la medida establecida en el art. 12, que permite compensar la energía eléctrica producida por las CCEE en las plantas de fuentes de energía renovables o de cogeneración de energía eléctrica de alta eficiencia o plantas híbridas, con la electricidad total consumida en las instalaciones de los socios de la CE y demás consumidores vulnerables que viven debajo de la línea de pobreza dentro de la región en la que se encuentra la sede de la CE.

\subsection{Problemática que plantea la naturaleza jurídica de la comunidad energética}

El legislador griego ha optado por configurar las CCEE como cooperativas, pero en cambio no exige que estas tengan fines mutualistas, sino que, como hemos visto, concibe las CCEE como organizaciones que persiguen principalmente fines de interés general. Las cooperativas con fines de interés general están previstas en Italia, Francia, Reino Unido, pero también en Grecia. De hecho, tanto las cooperativas sociales de responsabilidad limitada como las empresas sociales cooperativas a las que hicimos referencia en el apartado 3, son cooperativas que persiguen principalmente fines de interés general.

Pero no es sólo este aspecto el que llama la atención en relación con la identidad cooperativa, otros aspectos del régimen previsto para las CCEE ponen en duda su compatibilidad con los valores y principios cooperativos de la ACI; en particular con el principio de participación económica. Así cabe destacar las normas que regulan la distribución de los excedentes de la CE a sus socios sin diferenciar la procedencia de aquellos; o las que regulan la distribución del haber social durante la liquidación de la CE y que exige que, en muchos casos, esa distribución se haga en favor de los socios y en proporción a su participación en el capital social.

Si tenemos en cuenta el amplio abanico de actividades que pueden realizar las CCEE, tanto en interés de sus socios como no; la posibilidad de repartir los excedentes (o beneficios) entre aquellos, incluso en proporción al capital aportado, y los privilegios que la ley le reconoce como productor de energía, dudamos que todo ello sea compatible, no sólo con el estatuto de cooperativa sino también con la exclusividad que otorga a ésta como única forma jurídica de una comunidad energética tal y como prevé la legislación europea vista en el apartado 4.1.

\section{Modelos de Comunidades Energéticas en Grecia}

Atendiendo a como se configura la comunidad energética en la normativa analizada, podemos señalar algunos modelos de comunidades que podrían constituirse; para ello tomaremos en consideración, tanto la tipología de miembros participantes, como la actividad a desarrollar, y la ubicación que podrían tener.

Tabla. 2. Modalidades de comunidades energéticas

\begin{tabular}{|l|l|l|l|}
\hline $\begin{array}{l}\text { MIEMBROS DE LA COMUNIDAD } \\
\text { ENERGETICA }\end{array}$ & APLICACIÓN/ACTIVIDAD & REGIÓN \\
\hline VECINOS DE UN EDIFICIO DE VIVIENDAS & $\begin{array}{l}\text { COMPENSAR ENERGÍA } \\
\text { CONSUMIDA/PRODUCIDA }\end{array}$ & ÁREA URBANA \\
\hline
\end{tabular}


Los propietarios o usuarios de los apartamentos de un edificio crean una comunidad energética para instalar un sistema fotovoltaico o una turbina eólica en una propiedad que incluso puede estar ubicada en otra área y aplicar una compensación virtual entre la electricidad consumida en los apartamentos y la electricidad producida. De esta forma, los propietarios o usuarios de los apartamentos, miembros de la C.E, obtendrán una reducción en la factura de la electricidad.

Potencia indicativa: $50 \mathrm{~kW}$

Presupuesto indicativo: $€ 60,000$

Motivos: compensación virtual de la energía consumida por los miembros de la C.E.

Fines: No lucrativos

\section{VECINOS DE UN EDIFICIO DE VIVIENDAS}

\section{PROGRAMA DE ACTUALIZACIÓN DE LA ENERGÍA}

ÁREA URBANA

Los propietarios o usuarios de los apartamentos de un edificio crean una comunidad energética para participar en un programa de actualización de energía del edificio.

Presupuesto indicativo: $100.000 €$

Subvención indicativa: $50 \%$

Motivos: participación de la C.E. en programas

Fines: No lucrativos

\section{EMPRESARIOS}

\section{COMPENSAR ENERGÍA} CONSUMIDA/PRODUCIDA
ÁREA TURISTICA ISLA

Cinco empresarios (hoteles, etc.) establecen una comunidad energética para instalar un sistema fotovoltaico o una turbina eólica en una propiedad que incluso podría estar ubicada en otra área y aplicar una compensación virtual entre la energía consumida por las empresas, y la electricidad generada, consiguiendo así reducir los costos de la energía.

Potencia indicativa: $500 \mathrm{~kW}$

Presupuesto indicativo: $€ 500.000$

Motivos: compensación virtual de energía consumida por los miembros de la C.E.

Fines: No lucrativos

\section{AUTORIDADES LOCALES}

ACCIONES EN FAVOR DE
CONSUMIDORES
VULNERABLES/EVALUADOS

MUNICIPIO URBANO / PROVINCIAL

Tres autoridades locales crean una comunidad energética para instalar un sistema fotovoltaico o una turbina eólica y aplicar la compensación virtual entre la electricidad producida y la consumida por ellas y por personas vulnerables o ciudadanos que viven por debajo de la línea de pobreza.

Motivos: Disposiciones favorables de la ley para la participación de las autoridades locales, en acciones sobre consumidores vulnerables y compensación virtual de energía.

Fines: No lucrativos

\section{RESIDENTES - EMPRESARIOS - MUNICIPIO CALEFACCIÓN CENTRALIZADA}

Los residentes o empresarios de un área, junto con la municipalidad o la unión de empresas municipales de suministro de agua local, crean una comunidad energética, para instalar una unidad de producción de energía térmica con un sistema de calefacción de distrito para satisfacer sus necesidades de calefacción. Para la implementación del proyecto, podrán buscar fondos del Acuerdo Corporativo para el Marco de Desarrollo (ESPA) o de la Ley de Desarrollo.

Presupuesto indicativo: 5 millones $€$

Motivos: motivos generales de una C.E. (forma legal, participación en programas de financiación)

Fines: No lucrativos / lucrativos

\section{AGRICULTORES, SILVICULTORES, EMPRESARIOS}

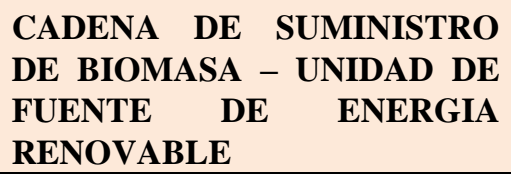

(ASENTAMIENTO DE MONTAÑAS, ÁREA AGRÍCOLA)

Los agricultores, los silvicultores y los empresarios que operan en el sector agrícola y forestal pueden constituir una comunidad energética con el objetivo de crear y administrar una cadena de suministro de biomasa y/o la instalación y operación de una unidad de biomasa de producción de energía eléctrica y térmica. La cadena de suministro puede suministrar la unidad de biomasa y productos como madera o pellets a los residentes, etc.

Motivos: motivos generales de una C.E. (forma legal, participación en programas de financiación). Incentivos especiales para unidades de fuentes de energía renovables (prioridad, garantías reducidas, 
exención de derechos de licencia, etc.)

Fines: Lucrativos

\section{AGRICULTORES/ EMPRESARIOS}

\section{INVERNADERO CON UNIDAD DE GENERACION DE ENERGIA Y CALEFACCION}

ÁREA AGRÍCOLA AREA AGRICOLA

Un grupo de agricultores o incluso empresarios agrícolas constituyen una comunidad energética para instalar una cooperativa de electricidad de alto rendimiento en un área donde pasa una red de gas. La electricidad generada se inyectará en la red y la energía térmica cubrirá las necesidades de un invernadero.

Presupuesto indicativo: 4 millones $€$

Motivos: motivos generales de una C.E. (forma legal, participación en programas de financiación). Incentivos especiales para unidades de fuentes de energía renovables (prioridad, garantías reducidas, exención de derechos de licencia, etc.)

Fines: Lucrativos

\section{RESIDENTES/EMPRESARIOS/MUNICIPIO}

\section{PLANTA DESALADORA CON FUENTES DE ENERGÍA RENOVABLES}

Los residentes y los empresarios de una isla pueden crear una C.E. con la participación del municipio, para implementar una planta de desalinización con fuentes de energía renovable (por ejemplo, con una turbina eólica), para abordar el problema de la escasez de agua.

Presupuesto indicativo: 2 millones $€$

Motivos: motivos generales de una C.E. (forma legal, participación en programas de financiación). Incentivos especiales para unidades de fuentes de energía renovables (prioridad, garantías reducidas, exención de derechos de licencia, etc.)

Fines: Lucrativos y/o no lucrativos, dependiendo de la participación de autoridades locales/privados

\section{RESIDENTES/EMPRESARIOS/MUNICIPIO}

\section{SISTEMA COMPLETO CON ALMACENAMIENTO}

Los residentes y los empresarios de una pequeña isla no conectada pueden establecer una comunidad energética con la participación de la municipalidad, cámaras, etc. para instalar un sistema de fuentes de energía renovable completo, con un sistema de almacenamiento para la gestión de la electricidad en el sistema autónomo.

Motivos: motivos generales de una C.E. (forma legal, participación en programas de financiación). Incentivos especiales para unidades de fuentes de energía renovables (prioridad, garantías reducidas, exención de derechos de licencia, etc.)

Fines: Lucrativos

\section{RESIDENTES/EMPRESARIOS/AUTORIDADES LOCALES}

\section{PEQUEÑA OBRA HIDROELÉCTRICA}

Los residentes o empresarios de un área junto con el municipio crean una comunidad energética, con el fin de instalar una pequeña obra hidroeléctrica de potencia indicativa de 1MW en el área

Motivos: motivos generales de una C.E. (forma legal, participación en programas de financiación). Incentivos especiales para unidades de fuentes de energía renovable (prioridad, garantías reducidas, exención de derechos de licencia, etc.)

Fines: Lucrativos

Fuente: www.naftemporiki.gr

\section{Experiencias de comunidades energéticas en Grecia}

La ley sobre Comunidades Energéticas nace en Grecia bajo un contexto de pobreza energética. En concreto, según los datos de la Autoridad Estadística Griega el porcentaje de hogares que declaran incapacidad financiera para tener calefacción adecuada en invierno es del $22.9 \%$, mientras que el porcentaje correspondiente para hogares pobres es del $40.8 \%$ y 4 de cada 10 hogares declaran incapacidad financiera para pagar las facturas de electricidad. 
El hecho de que los ingresos de la población se hayan reducido en un 30\%, junto con la volatilidad de los precios y los altos costos de la energía, se consideran factores decisivos que impiden cubrir adecuadamente las necesidades de consumo de energía de la población, especialmente para los grupos vulnerables, que son los más afectados (Douvitsa, 2018: 38).

Uno de los objetivos de la Ley 4513/2018 es justamente la lucha contra la pobreza energética, así como la promoción de la energía sostenible.

La Ley 3851/2010 que lleva por título "Acelerar el crecimiento de las fuentes renovables de energía para enfrentar el cambio climático y otras disposiciones sobre asuntos de competencia del Ministerio de Medio Ambiente, Energía y Cambio Climático", prevé que Grecia tiene como objetivo cubrir el 20\% de sus necesidades energéticas a través de fuentes de energía renovables en el año 2020 (artículo 1.3.a.).

Según la Autoridad Reguladora de la Energía (RAE), a finales de 2017 había una brecha energética de al menos 2,000MW, que debería cubrirse hasta 2020, para poder llegar al objetivo mencionado anteriormente. La creación de unidades de fuentes de energía renovables por parte de las comunidades energéticas (CC.EE) puede contribuir a alcanzar dicho objetivo.

Hasta la fecha, el número de las comunidades energéticas (CC.EE.) registradas en el Registro General Mercantil asciende a 330. De ellas 315 están activas, una está en fase de disolución/liquidación y 12 están en estado de preinscripción y se trata de comunidades energéticas con sede de actividad en Attica. Además, observamos 2 que están en la fase de eliminación y se registraron en la Cámara Mercantil e Industrial de Salónica.

Como se puede observar en la tabla siguiente, la gran mayoría de las Comunidades Energéticas se encuentran en el norte de Grecia, y en concreto en la región geográfica de Macedonia y de Tracia (58\%) mientras alrededor de un 20\% de ellas están activas en la región de Grecia Central.

Tabla. 3. Número de Comunidades Energéticas por Región Geográfica

\begin{tabular}{|l|l|}
\hline \multicolumn{2}{|c|}{$\mathbf{N}^{\mathbf{0}}$ de Comunidades Energéticas por Región Geográfica } \\
\hline Creta & 2 \\
\hline Epiro & 23 \\
\hline Grecia Central o Continental & 70 \\
\hline Macedonia & 156 \\
\hline Peloponeso & 13 \\
\hline Tesalia & 30 \\
\hline Tracia & 36 \\
\hline
\end{tabular}

Fuente: Elaboración propia basada en los datos del Registro

En el siguiente apartado se exponen algunos casos de comunidades energéticas en Grecia.

\subsection{Minoan Comunidad Energética}

"Minoan Energiaki" se creó por acuerdo de su asamblea constituyente a principios de octubre de 2019, de acuerdo con la Ley 4513/2018. Se trata de una cooperativa cuyo único propósito es promover la economía social y solidaria, la innovación en el sector energético y la lucha contra la pobreza energética, la producción, almacenamiento, autoconsumo, distribución y suministro de energía, a través de la actividad en los sectores de fuentes de energía renovables, coproducción de electricidad y calor, uso racional y eficiencia energética.

Según sus estatutos, la Comunidad Energética de Minoan desarrolla las siguientes actividades:

a) Producción, almacenamiento, autoconsumo, venta de energía eléctrica o térmica o de refrigeración de plantas de FER, coproducción de electricidad y calor de alto rendimiento, o plantas híbridas instaladas dentro de la Unidad Regional de Creta donde se encuentra la sede de la Comunidad Energética,

b) Gestión, como la recolección, el transporte, el procesamiento, el almacenamiento o la eliminación, de materia prima para la producción de energía eléctrica o térmica, o de enfriamiento, a partir de biomasa o biocombustibles o biogás, o mediante la utilización de energía de la fracción de desechos urbanos biodegradables, 
c) Suministro para miembros de productos, aparatos e instalaciones energéticos, con el objetivo de reducir el consumo de energía y el uso de combustibles convencionales, así como mejorar la eficiencia energética,

d) Suministro para miembros de vehículos eléctricos, híbridos o no, y vehículos en general que utilizan combustibles alternativos,

e) Distribución de electricidad dentro de la Unidad Regional donde se encuentra su sede,

f) Suministro de electricidad o gas natural a clientes finales, de conformidad con el artículo 2 de la ley 4001/2011 (A 179), dentro de la Unidad Regional donde se encuentra su sede,

g) Producción, distribución y suministro de energía térmica o de refrigeración dentro de la Unidad Regional donde se encuentra su sede,

h) Gestión de la demanda para reducir el uso final de la electricidad y representar a los productores y consumidores en el mercado de la electricidad,

i) Desarrollo de redes, gestión y explotación de infraestructuras de combustibles alternativos, de conformidad con la Ley 4439/2016 (A 222) o gestión de medios de transporte sostenible dentro de la Unidad Regional donde se encuentra la sede de la comunidad energética,

j) Prestación de servicios energéticos, de conformidad con el artículo 10 de la Decisión D6 /13280/ 7.6.2011 (B 1228) del Ministro de Medio Ambiente, Energía y Cambio Climático.

Son miembros de esta comunidad energética tanto personas físicas como empresarios, así como personas jurídicas de derecho público. Para ser miembro hay que aportar a capital una cuota de 100 euros, que es obligatoria. Por supuesto, quien quiera puede adquirir más acciones hasta el $20 \%$ del capital social. El número de miembros fundadores fue de 38 .

\subsection{Comunidad Energética de Karditsa}

En la ciudad de Karditsa, donde se ha desarrollado un ecosistema de empresas sociales (del sector productivo, bancario y de servicios) que está funcionando con éxito y que se apoyan mutuamente tanto compartiendo conocimientos como ofreciendo asistencia financiera, se encuentra la Sociedad Cooperativa Energética de Karditsa. Se trata de una cooperativa civil que se formó en 2010 de acuerdo con la Ley 1667/1986 y en ella participan 400 miembros.

Desde 2016, la Sociedad Cooperativa Energética de Karditsa ha completado el proyecto para la creación de una planta para la producción de biocombustibles sólidos en el área de Karditsa. La fábrica produce 1.100 toneladas de pellets de madera de alta calidad. La materia prima para la producción de los agregados proviene de residuos de biomasa forestal y agrícola, así como piezas de madera o aserrín de las industrias locales. Según sus estatutos, el objetivo principal de la cooperativa es crear una unidad o unidades de energía, una vez se completen los procedimientos y se obtenga el permiso correspondiente requerido de la Autoridad Reguladora de Energía.

En abril 2018 la asamblea general de la Cooperativa decidió la modificación de sus estatutos de acuerdo con la Ley 4513/2018, y así, según el artículo 9 párrafo 6 la Cooperativa se convierte a Comunidad Energética bajo el nombre de "Comunidad Energética de Karditsa Syn.P.E5"

\subsection{Comunidad Energética de Tesalia}

La primera comunidad cnergética agrícola en Grecia se creó en la región de Tesalia, tiene su sede en Larissa y es autofinanciada. La idea fue implementada por el Ministerio de Desarrollo Rural y Alimentación y organismos locales de la región que representan a más de 25000 agricultores. El único propósito de la primera comunidad energética agrícola es reducir los costos de energía hasta en un $80 \%$ para el riego de los cultivos en los próximos años (Papazafeiropoulos, 2019, p.78).

Cabe señalar que en esta comunidad energética se instala la primera turbina eólica griega EW 16 Thetis del grupo EUNICE con una capacidad de $50 \mathrm{~kW}$ y un parque con una potencia fotovoltaica de $950 \mathrm{~kW}$.

En la primera fase, con la instalación del sistema fotovoltaico, se cubrirán las necesidades de más de 5.000 agricultores con el objetivo de expandirse más adelante y satisfacer todas las necesidades de mejoras de tierras de Tesalia, es decir, alrededor de 25.000 agricultores. Esto supone un ahorro de más de 2 millones de euros en un período de 20 años, que los agricultores pagarían por su energía. Es decir, estamos hablando de un beneficio financiero anual de más de $€ 70-80.000$, que será transferido a los agricultores en las facturas de electricidad.

\subsection{Cooperativa Energética de la Isla de Sifnos}


La Cooperativa de Sifnos ${ }^{6}$ fue creada en 2013 bajo el nombre de "Cooperativa Energética y de Desarrollo de Sifnos" con el fin de satisfacer las necesidades de sus miembros en áreas como la energía verde, la protección del medio ambiente, el transporte. Actualmente, tiene más de 100 miembros, residentes permanentes de la isla y visitantes.

De acuerdo con sus estatutos, el propósito de la cooperativa es organizar:

a) La producción y disposición de la energía producida por cualquier fuente renovable y el potencial total de la isla.

b) La producción, gestión, procesamiento y distribución de los productos y servicios de la isla.

c) La promoción de soluciones y actividades socialmente responsables en Sifnos.

El proyecto, que la cooperativa pretende llevar a cabo, es una planta de energía híbrida que consiste en un parque eólico y una planta hidroeléctrica reversible. Podrá satisfacer todas las necesidades energéticas de Sifnos, utilizando solo fuentes de energía renovable. Las instalaciones se basarán en la protección del medio ambiente y el producto turístico de la isla.

En concreto, el proyecto consiste en un pequeño parque eólico de 5 turbinas eólicas, un lago artificial sellado de más de 1,000,000 de metros cúbicos, excavado en un área rocosa cerca del mar a una altitud de 330 metros, una central hidroeléctrica con 4 turbinas hidráulicas y una estación de bombeo con 12 bombas.

La electricidad generada por las turbinas eólicas se entregará al mismo tiempo a la red y las bombas que elevarán el agua de mar, llenando el lago artificial. El proyecto hidroeléctrico operará permanentemente, proporcionando estabilidad de red. Si la producción de electricidad del parque eólico no es suficiente para el suministro seguro de la red, el proyecto hidroeléctrico producirá la energía restante. Incluso en el caso de varios días de apnea, la planta hidroeléctrica podrá suministrar electricidad a la isla sin ningún problema.

Para lograr su objetivo, la Cooperativa de Sifnos firmó un acuerdo de cooperación con el Municipio de Sifnos y se convirtió en miembro de la Federación Europea de Cooperativas de Energía de Fuentes de Energía Renovables REScoop.eu y al mismo tiempo, preparó un estudio sobre la progresiva autonomía $100 \%$ energética de Sifnos por fuentes de energía renovables y puso en marcha algunas acciones de desarrollo sostenible de menor alcance, como la acción para reducir el uso de bolsas de plástico en la isla.

En la asamblea general ordinaria anual de los miembros de la cooperativa celebrada el 18 de febrero de 2018, se decidió por unanimidad transformar la Cooperativa Energética de Sifnos en una Comunidad Energética de acuerdo con las disposiciones de la ley 4513/2018, para facilitar la entrada de nuevos miembros y la participación de la Municipalidad de Sifnos.

\section{Financiación de las comunidades energéticas}

Según se indica en la página web del Servicio Especial de Gestión del Programa Operativo "Competitividad y Emprendimiento", la acción "Impulso del establecimiento y la operación de Comunidades Energéticas" está cofinanciada por el Fondo Europeo de Desarrollo Regional (FEDER) en el marco del Programa

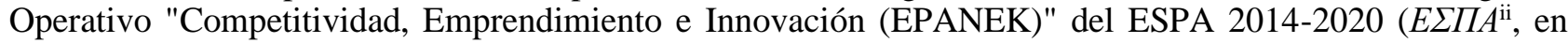
griego).

Cada comunidad energética presentará una única solicitud de financiación vinculada con esta acción.

Las actividades elegibles que financiará la acción son las siguientes:

- Generación de electricidad a partir de la conversión de energía eólica.

- Generación de electricidad a partir de la conversión de energía solar.

- $\quad$ Generación de electricidad a partir de plantas generadoras de energía y calor.

- $\quad$ Producción de electricidad a partir de centrales hidroeléctricas.

- $\quad$ Servicios de generación de electricidad.

- Servicios de generación de electricidad a partir de sistemas fotovoltaicos para suministro de vapor y agua caliente, para calefacción urbana.

Servicios de suministro de vapor y agua caliente a través de tuberías de distribución de suministro.

- $\quad$ Servicios de desalinización de agua de mar.

Según la información del Ministerio de Medio Ambiente y Energía, las ayudas a las comunidades energéticas estarán en el rango del $40 \%$ en promedio (subsidio del costo del proyecto), mientras se espera que el límite máximo de la financiación alcance 1 millón de euros por plan de inversión.

\section{Conclusiones}


1. Grecia viene impulsando la economía social en los últimos años, con diversas leyes y políticas, pero a diferencia de otros países, el concepto de economía social se circunscribe fundamentalmente a las cooperativas, y no a todas. Para considerarse entidad de la economía social es necesario que sus actividades generen un beneficio común o social; sus decisiones se adopten democráticamente; los beneficios no se distribuyan entre los socios (salvo que sean trabajadores); que la horquilla no supere tres veces el salario mínimo; que cooperen con otras entidades de la economía social y solidaria, y que no dependan directa o indirectamente de entidades públicas. Características todas ellas que recuerdan a los principios cooperativos de la Alianza Cooperativa Internacional.

2. Entre otras leyes, Grecia aprobó en 2018 la Ley sobre Comunidades Energéticas, con la que se regula por vez primera en Europa esta forma de organización con la que se quiere promover la transición a las energías renovables, hacer frente a la pobreza energética y dar más protagonismo a los consumidores (ciudadanos) en la generación, distribución, almacenamiento y comercialización de estas energías. La regulación griega de las comunidades energéticas no dista mucho de las directivas aprobadas por las instituciones europeas (2018/2001) y (2019/944), salvo por el hecho de que éstas no condicionan la forma jurídica que pueda tener una comunidad energética, siempre que tenga personalidad jurídica; se base en la participación abierta y voluntaria de sus miembros; sea autónoma; sus miembros, personas físicas o jurídicas, públicas o privadas, deben tener vinculación con el territorio donde se desarrolla el proyecto energético, y ninguno de ellos pueda tener por sí solo el control de la comunidad.

3. Grecia en cambio, ha conformado la comunidad energética como una cooperativa civil, regida por tanto por la ley 1667/1986, pero acreedora de medidas de fomento como las empresas sociales cooperativas sometidas a la Ley 4399/2016. La regulación de la comunidad energética no impide que se sigan constituyendo cooperativas energéticas como cooperativas civiles, sociales o de trabajadores, conforme a sus respectivas leyes. La Ley de Comunidades Energéticas acota el objeto social que pueden tener estas, no permitiendo ampliar el mismo a otras necesidades más allá de las expresamente previstas en la Ley.

4. Del análisis de esta ley hemos destacado la finalidad social de la comunidad, más que mutualista; la posible presencia como miembro de las entidades públicas y empresas públicas, lo cual es una novedad en Grecia; el voto único por persona; la no distinción de resultados del ejercicio y su posible reparto entre los miembros, e incluso, en el momento de la liquidación se contempla el reparto del saldo resultante entre los miembros en proporción al capital aportado por cada uno. Como hemos visto, la doctrina griega ha criticado que se exija la forma jurídica de cooperativa para la comunidad energética, y que se someta la misma a un régimen jurídico extraño a la cooperativa, en el que ni siquiera se exige la inclusión de la forma jurídica cooperativa en su denominación, ni su inscripción en el Registro de Cooperativas.

5. Dejando a un lado la problemática jurídica que plantea la regulación de las comunidades energéticas en Grecia, hemos analizado las posibilidades que esta nueva figura puede ofrecer para atender necesidades específicas que pueden plantearse. Hemos presentado 10 modelos de comunidades energéticas que pueden dar respuesta a diversos intereses, desde una comunidad de vecinos que busca el suministro de energía renovable y puede compensar la energía consumida con la energía producida; una comunidad promovida por autoridades locales que buscan dar servicio energético a todos los habitantes, con independencia de su capacidad económica; una comunidad formada por agricultores/silvicultores para producir energía a partir de biomasa, o los residentes de una isla que crean una comunidad para abastecerse de energía en sus hogares y empresas y proporcionar energía renovable a una planta de desalinización que les garantice también agua potable.

6. No hemos querido concluir el trabajo sin conocer qué experiencias se han llevado a cabo hasta el momento en Grecia bajo la fórmula de comunidad energética. De la información ofrecida por las instituciones públicas helenas, y la difundida por las propias iniciativas, hemos comprobado que existen 330 comunidades energéticas de las cuales 315 son activas, 12 están en proceso de inscripción y 3 en proceso de liquidación. De todas ellas, hemos seleccionado cuatro experiencias para conocer más a fondo cómo se han organizado y qué intereses están atendiendo.

7. Del análisis de las comunidades energéticas seleccionadas se observa el importante papel que la administración pública puede jugar, desde promover una $\mathrm{CE}$ en favor de todos los agricultores de la zona (Tesalia), a participar en proyectos que buscan garantizar el suministro energético en zonas de isla (Creta o Sifnos). También observamos como la comunidad energética puede contribuir a promover nuevas actividades económicas (producción de pellets en Karditsa) e incentiva la innovación técnica, como la turbina eólica Thetis de fabricación griega.

8. Todos estos proyectos han sido posibles gracias y principalmente a la existencia de políticas públicas que han contribuido a su promoción. Estas políticas han sido generadas a nivel estatal y local, pero, sobre todo, han contado con el apoyo de las Instituciones Europeas (FEDER), porque no olvidemos que la transición energética a nuevas fuentes de energía renovable es una prioridad para Europa. España debería implementar lo antes posible las directivas europeas que hemos comentado en este trabajo para propiciar la constitución de comunidades energéticas y facilitar el acceso de los emprendedores a la financiación existente. Recientemente, mediante la Disposición final sexta del Real Decreto-Ley 23/2020, de 23 de junio 
de medidas en materia de energía, se ha declarado la incorporación parcial de la Directiva (UE) 2018/2001, pero la norma no despeja todas las dudas que plantea este modelo por lo que habrá que esperar a su desarrollo. España como Grecia son países con difícil acceso a las redes eléctricas internacionales por su ubicación periférica, pero en cambio tienen un gran potencial como generadores de energías renovables, procedan del sol, del viento o del mar. España no puede renunciar a estar en primera línea en este proceso de tránsito.

\section{Referencias bibliográficas}

Avarlis, D. (2018) Cooperativa energética de Karditsa: un proyecto que ha sido adoptado por la sociedad. Recuperado de https://www.energia.gr/article/124894/energeiakh-synetairistikh-etaireia-karditsas-ena-egheirhma-poy-eheiagkaliastei-apo-thn-koinonia.

Autoridad Estadística Griega. (s.f.) Recuperado de https://www.statistics.gr/el/home.

Douvitsa, I. (2018) The new law on energy communities in Greece. Cooperativismo e Economía Social, № 40, pp. 3158. DOI: https://doi.org/10.35869/ces.v0i40.1385.

Drosou, O. (Ed.) (2019) Construyendo comunidades energéticas en Grecia. DOI: 978-618-81299-9-3.

Fajardo, G. (2019) New perspectives for the cooperativism of renewable energies: Legal recognition and promotion. International Journal of Cooperative Law Issue, II, pp. 111-126. Recuperado de www.iuscooperativum.org.

Fajardo, G., \& Frantzeskaki, M. (2018) Las comunidades energéticas en Grecia. Marco legal y compatibilidad con los fines y principios cooperativos. Comunicación presentada en el XVII Congreso Internacional de Investigadores en Economía Social. La Economía Social: Transformaciones Recientes, Tendencias y Retos de Futuro, Toledo.

Fajardo, G., \& Frantzeskaki, M. (2017) La economía social y solidaria en Grecia. Marco jurídico, entidades y principales características. REVESCO. Revista de Estudios Cooperativos, Tercer Cuatrimestre, No 125, pp. 49-88. DOI:10.5209/REVE.58135.

Fajardo, G. \& Talaverano, J. (2020) Prosumidores de energía renovable. Marco legal y buenas prácticas, en El potencial de los Prosumidores de energía renovable en la transición energética de la Ciudad de Valencia. Publicaciones UPV (en prensa).

Kaminari, O. \& Papageorgiou, K. (2016) Los conceptos de excedentes, beneficios e ingreso neto en cooperativas. Recuperado de http://isem-journal.blogspot.com/2016/04/blog-post_72.html.

Kataskevesktirion.gr. (2018) Un plan progresivo para la autonomía energética al 100\% de Sifnos [presentación del modelo de proyecto + video]. http://kataskevesktirion.gr/\%CE\%AD\%CE\%BD\%CE\%B1\%CF\% $80 \% \mathrm{CF} \% 81 \% \mathrm{CE} \% \mathrm{BF} \% \mathrm{CE} \% \mathrm{BF} \% \mathrm{CE} \% \mathrm{~B} 4 \% \mathrm{CE} \% \mathrm{~B} 5 \% \mathrm{CF} \% 85 \% \mathrm{CF} \% 84 \% \mathrm{CE} \% \mathrm{~B} 9 \mathrm{CE} \% \mathrm{BA} \% \mathrm{CF} \% 8 \mathrm{C}-$ \%CF\%83\%CF\%87\%CE\%AD\%CE\%B4\%CE\%B9\%CE\%BF-\%CE\%B3\%CE\%B9\%CE\%B1\%CF\%84\%CE\%B7\%CE\%BD-100-\%CE\%B5\%CE\%BD\%CE\%B5\%CF\%81\%CE\%B3/.

Minoan Energy Community. Recuperado el Mayo 23, 2020 de https://minoanenergy.com/.

Papazafeiropoulos, C. (2019) Comunidades energéticas: marco jurídico y estudio de implementación en Mesta, Chios (Tesis, Universidad el Egeo). Disponible en Repositorio Institucional HELLANICUS https://hellanicus.lib.aegean.gr/handle/11610/19364.

RESCoop (2020) Energy Communities under the Clean Energy Package. Transposition Guidance. Recuperado de https://uploads.strikinglycdn.com/files/48701cfd-f397-4903-9d361fba162223f4/Energy\%20Communities\%20Transposition\%20Guidance.pdf.

Servicio especial de gestión del Plan Empresarial Competitividad Emprendimiento Innovación (2020) Pre-publicación sobre la Invitación del Programa "Mejoramiento del establecimiento y funcionamiento de comunidades energéticas". Recuperado de http://www.antagonistikotita.gr/epanek/news.asp?id=750.

https://www.businessregistry.gr/publicity/index (datos consultados el mayo de 2020).

ii Acuerdo Corporativo para el Marco de Desarrollo, es el principal plan estratégico para el desarrollo del país con la ayuda de importantes recursos provenientes de los Fondos Estructurales y de Inversión de la Unión Europea. 